\title{
Concurso Nuevo Plan Director PUC / Alameda - Lira
}

El Jurado estuvo compuesto por: Pedro Bannen, arquitecto (presidió en representación del Rector); Miguel Baudizzone, arquitecto argentino; Víctor Gubbins, arquitecto, Premio Nacional de Arquitectura; Alejandro Gutiérrez, arquitecto (representante de los concursantes); Alberto Dittborn, Director de la Escuela de Diseño de la PUC; Hans Mühr, Director de Infraestructura de la PUC; Alfonso Swett, empresario. El Director del Concurso fue Luis Eduardo Bresciani P., arquitecto; y actuaron como asesores Ricardo Labarca y Tomás Dalla Porta, arquitectos. La Pontificia Universidad Católica de Chile invitó a un grupo de arquitectos a contribuir a su futuro desarrollo institucional a través de propuestas para un Plan Director en los terrenos que la Universidad posee en la manzana adyacente al Campus de la Casa Central, hacia el poniente por la Avda. Bernardo O'Higgins.

El estudio pedido se inscribía en un continuo histórico muy significativo que ha caracterizado a nuestra Universidad. El Plan de Desarrollo de la Pontificia Universidad Católica de Chile, presentado en enero del 2001, señala:

"Planificar el desarrollo inmobiliario de los bienes raíces destinado al patrimonio de reserva y su conversión progresiva hacia una cartera de inversiones en activos financieros, y gestionar la realización de proyectos corporativos que incrementen el patrimonio general de la Universidad".

\section{Carácter del encargo}

Se resolvió invitar a un pequeño grupo de oficinas de arquitectos. Desde el punto de vista de un estudio de prefactibilidad económica realizado, se señaló que los principales beneficios que debería aportar un Plan Director para el terreno mencionado eran:

1. Permitir generar rentas efectivas para terceros, en un terreno que no deja de ser propiedad de la Universidad.

2. Dotar a la Universidad de un espacio físico que le permita consolidar sus actuales ventajas comparativas frente a una creciente competencia.

3. Posibilitar la liberación de algunos espacios de la Casa Central actual para dedicarlos a usos académicos directos, como así mismo repensar la localización de programas de extensión universitaria.

\section{Premisas arquitectónicas}

1. Conexión funcional con campus Casa Central: considerar un vínculo funcional continuo entre el campus y el terreno materia del encargo.

2. Presencia pública de la PUC en la Alameda: el terreno de Lira con la Alameda constituye una importante oportunidad. Los actuales $160 \mathrm{~m}$ que ocupa la Casa Central frente a la Alameda se extienden a $240 \mathrm{~m}$.

3. Sustentabilidad en el tiempo del proyecto: aquí se consignan las articulaciones programáticas que garantizarían un modo de ocupación a través del tiempo. No se contaba con un programa detallado sobre las necesidades a cumplir en el terreno de Lira - Alameda - Quito, por lo tanto, la propuesta debía considerar grandes unidades programáticas:

- Estacionamientos: $20.000 \mathrm{~m}^{2}$ (para 700)

- Recintos universitarios: $15.000 \mathrm{~m}^{2}$ aprox.

- Hotel: $5.000 \mathrm{~m}^{2}$ aprox.

- Oficinas: $30.000 \mathrm{~m}^{2}$ aprox.

- Total: $70.000 \mathrm{~m}^{2}$ aprox.

4. Estando en juego la imagen pública de la PUC, cada una de las etapas debía ser completa en sí misma.

5. Flexibilidad: una de las características más relevantes del actual edificio de la Casa Central es acoger en sus espacios construidos los más diversos programas.

6. Ciudad: del momento que esta operación se hace de cara a la ciudad, lo que se proponga debe abordarse también desde la ciudad que le da origen.

7. Etapas de implementación: dado el importante número de metros cuadrados a construir y la compleja gestión que implica cada una de las unidades programáticas señaladas, la estrategia para llevar adelante este Plan Director requería establecer etapas en el tiempo para su implementación. Debían considerarse tres etapas para la implementación total del Plan Director. Era responsabilidad de los arquitectos establecer cómo se combinaban las distintas unidades programáticas producto de la subdivisión de los cuatro grandes programas enunciados y así configurar las tres etapas contempladas. Cada etapa no podía depender de la implementación de otra etapa. Que ello sea posible sería uno de los aspectos a evaluar en las proposiciones.

\section{Primer premio}

\section{fuan Ignacio Baixas y Enrique Del Río}

El proyecto genera tres elementos arquitectónicos: un patio urbano, edificios continuos y una torre de $100 \mathrm{~m}$.

a. Patio urbano: Se propone 'un cuarto' patio del campus, prolongando su frente a la Alameda. Este patio se lee como un vacío urbano y se genera en una prolongación del nivel de piso noble de la universidad hacia el poniente el cual, gracias a la pendiente de Santiago, pasa por sobre la calle Lira y permite una continuidad peatonal interior. Este cuarto patio nace de la prolongación de los corredores actuales de la universidad; a su vez el patio es el remate de una diagonal incipiente que nace de la esquina opuesta de Marcoleta y Portugal. Bajo el patio se propone un salón-zócalo, complementario a las actividades de extensión; en suma, un espacio público de mucho interés paisajístico y de uso, con un control total por parte de la PUC, y además permite un plan de etapas variado y de gran flexibilidad.

b. Edificios continuos: Se propone una fachada continua de $35 \mathrm{~m}$ de altura para conformar el perímetro edificado del patio. Su expresión sería de muros perforados y se propone rematarlos con terrazas-jardín, estableciendo un nuevo vínculo con el Cerro Santa Lucía y sus terrazas.

c. Torre: Este tercer elemento integraría la sede universitaria a la escala actual del centro de la ciudad, planteando en el skyline una unificación con las torres ubicadas en la Remodelación San Borja.

\section{Segundo premio}

Rodrigo Pérez de Arce, Sebastián Bianchi, Patricio Mardones y Carolina Portugueis

Cualquier opción de proyecto quedaba sometida al forcejeo propio del requerimiento excesivo de espacio construido y su calce en el esquema de rasantes y cabidas derivado de la normativa: había que meter con prestancia un elefante en la tina de baño (pensamos que el rigor de las rasantes fue -al menos parcialmente- desestimado por el jurado). Tal fricción no es el mas estimulante campo de desarrollo de la arquitectura y sus valores : basta ver lo que ha ocurrido en las ciudades de Chile. Por momentos nos parecía que este forcejeo de cabidas era perfectamente inútil y estuvimos a punto de abandonar la empresa.

La invención de la torre doble (pensábamos en el interés que tiene la torre de los ministerios en Brasilia) junto a la posibilidad de desarrollarla como un fuste de columna, libre de las hinchazones y taras propias del aludido paisaje de rasantes, nos convenció que existía al menos ese camino digno y propositivo. De paso nos permitía responder a los complejos requerimientos de desarrollo por etapas (cada torre era completa en si misma). El vacío central de la torre contarrestaba esa cualidad orillera de las edificaciones del flanco sur de la Alameda, cualidad de pandereta urbana de gran tamaño causante de conos de sombra, aislamiento y un caos del abandono de las manzanas situadas hacia el sur.

Pensábamos que la Universidad Católica debía asumir ese enlace con la ciudad en todos sus 
frentes; mas aún ahora que con este proyecto, abandonada la vieja modalidad de UniversidadClaustro se iniciaba la nueva fase de UniversidadAbierta. Situado sobre el túnel vehicular de Lira, un cuarto patio señalaba esa nueva dimensión, en secuencia con los patios claustro de la Casa Central pero comportándose como terraza urbana expuesta y accesible desde la calle.

\section{Tercer premio}

Eduardo San Martín, Pedro Gastón Pascal y Andrés Mariaschi

Resultan interesantes algunos criterios básicos de la propuesta como la consolidación de un claustro interno semi-cubierto en el eje de la Casa Central, el diseño de la fachada unificando con la misma, y la apertura de una callejuela entre Lira y Quito. Esta última decisión tiene -al mismo tiempo- efectos negativos sobre la propuesta: separa el volumen a construirse sobre calle Lira al sur del resto del desarrollo y deja el deslinde sur del predio con la fachada inconclusa del edificio existente.
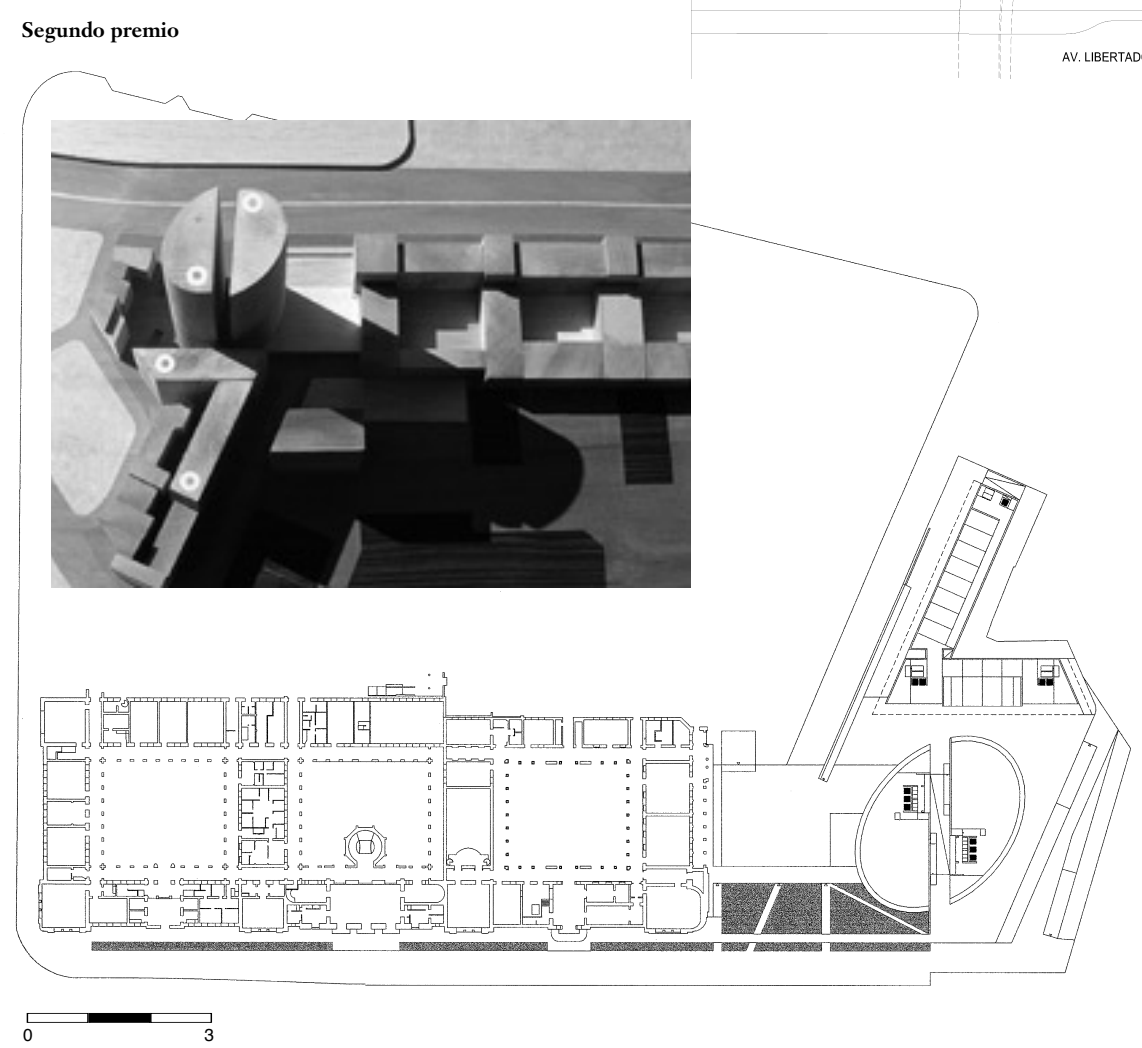
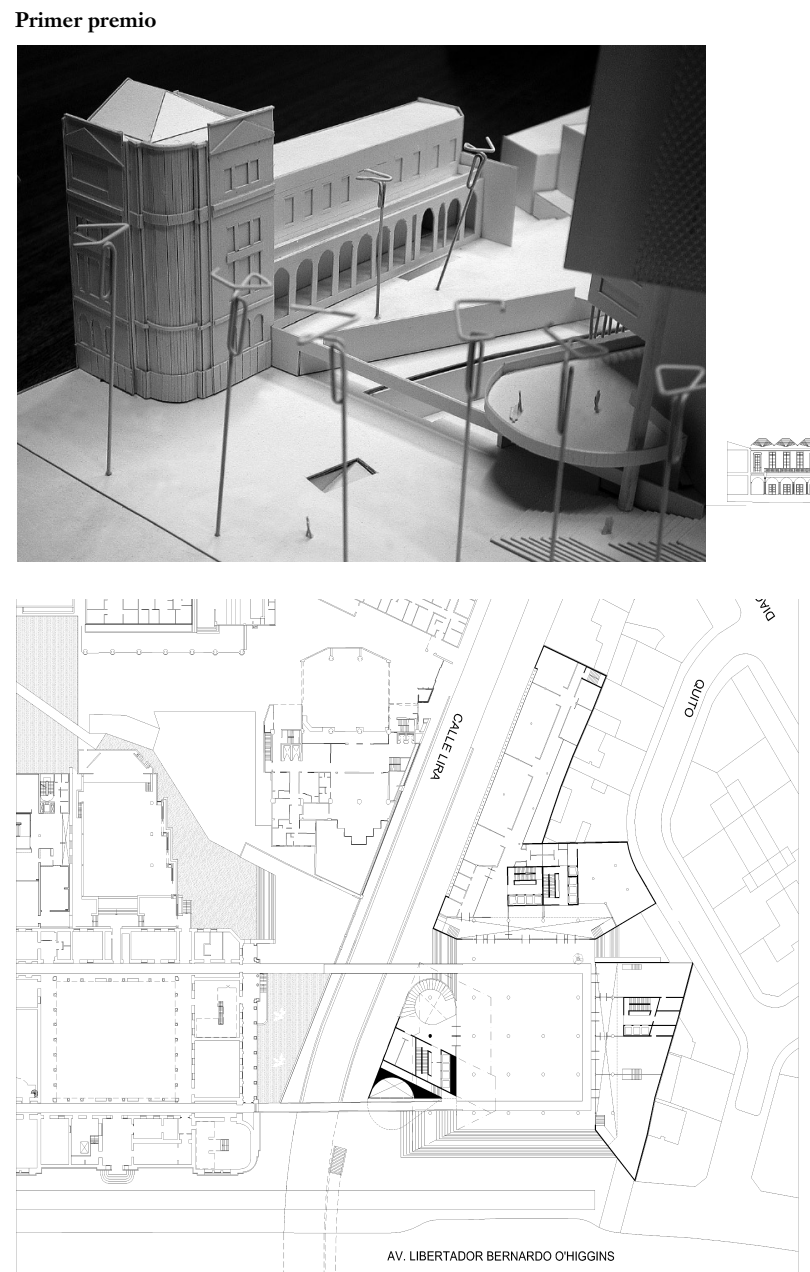

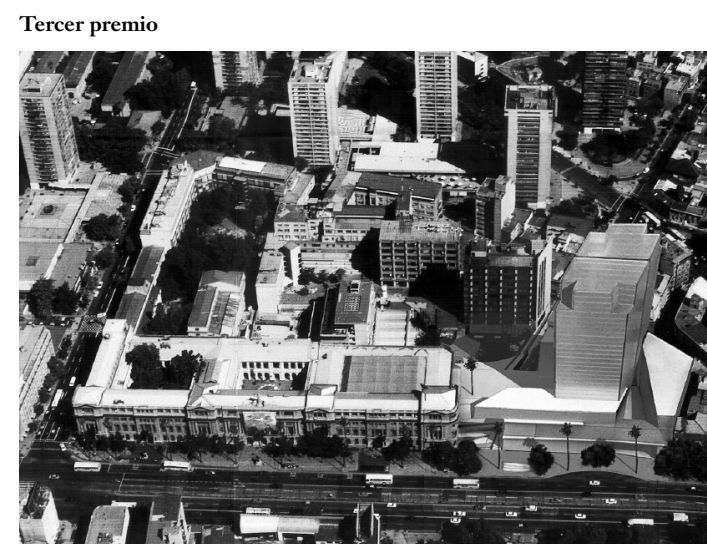

